\title{
CALIDAD DE VIDA FAMILIAR Y BIENESTAR SUBJETIVO EN JÓVENES CON DISCAPACIDAD INTELECTUAL DE UN ESTABLECIMIENTO CON EDUCACIÓN ESPECIAL Y LABORAL DE LA CIUDAD DE TALCA*
}

\section{QUALITY OF FAMILY LIFE AND SUBJECTIVE WELL-BEING IN YOUNG PEOPLE WITH INTELLECTUAL DISABILITY IN SCHOOL WITH SPECIAL EDUCATION OF THE TALCA CITY}

\author{
Yohanina Andrea Muñoz Valdés**, Yohanna del Pilar Poblete Toloza ${ }^{* *}$ y Andrés \\ EdUARDO JIMÉNEZ FIGUEROA***
}

\begin{abstract}
*Trabajo realizado en el marco del Programa de Investigación Calidad de vida y ambientes saludables (DPI, Universidad de Talca, 2007).

**Psicóloga Clínica. Asistente del Programa de Investigación Calidad de vida y ambientes saludables . E-Mail: yohanina.munoz@gmail.com

***MA en Psicología Social y Psicóloga Organizacional. Asistente del Programa de Investigación Calidad de vida y ambientes saludables de la Facultad de Psicología, Universidad de Talca. E-Mail: ypoblete@alumnos.utalca.cl

****MA en Administración y Dirección de Recursos Humanos y Psicólogo Organizacional. Docente de la Facultad de Psicología de la Universidad de Talca. E-Mail: anjimenez@utalca.cl
\end{abstract}

\section{REsUMEN}

El estudio que se presenta tuvo como objetivo identificar la relación entre la Calidad de Vida Familiar, Funcionamiento Familiar y Bienestar Subjetivo junto con los factores de la calidad de vida familiar en relación al bienestar subjetivo en jóvenes con discapacidad intelectual, alumnos de un establecimiento de educación especial y laboral de la ciudad de Talca (Chile). La muestra fue dividida en dos grupos: uno de ellos estuvo integrado por 53 alumnos de 16 a 24 años de edad, del citado establecimiento que fueron diagnosticados con discapacidad intelectual: el $52.8 \%$ de ellos manifiesta discapacidad intelectual como único predictor de su condición, mientras que el $47.2 \%$ restante corresponde a comorbilidad de ésta con otros tipos de discapacidad asociados. Los niveles de discapacidad van desde el leve (37.7\%), seguido por el moderado (50.9\%) y finalmente el severo y el profundo (3.8\%). El otro grupo estuvo conformado por 53 apoderados, padres o tutores del mismo establecimiento educacional.

Con la finalidad de medir las variables de estudio se administraron cuatro instrumentos: (1) la Escala de Calidad de Vida Familiar (Verdugo, Sainz \& Rodríguez, 2009), (2) la versión traducida al español (Bellon, Delgado, Luna del Castillo \& Lardelli, 1996) del Family Functioning Questionnaire (Smilkstein, Ashworth \& Montano, 1982), (3) la adaptación y validación de Moyano y Ramos Alvarado (2007) de la Satisfaction with the Life Scale (Diener, Emmons, Larden \& Griffin) y (4) la versión chilena de Moyano-Díaz \& Ramos (2007) de la Subjective Happiness Scale (Lyubomirsky \& Lepper, 1999). Para realizar el estudio se utilizó una metodología descriptiva y correlacional de corte transversal a fin de establecer relaciones de concomitancia entre las variables. Los resultados principales demostraron una relación significativa entre im- 
portancia de la calidad de vida familiar y satisfacción de la calidad de vida familiar $(r=.402$; $p<.01)$; entre felicidad subjetiva y satisfacción vital $(r=.457 ; p<.01)$ y entre felicidad subjetiva $\mathrm{y}$ funcionamiento familiar $(r=.388 ; p<.05)$.

Los siguientes factores de la calidad de vida familiar percibidos por los padres: interacción familiar, rol parental, bienestar físico y material, bienestar emocional y apoyo a las personas con discapacidad no mantuvieron relación con la felicidad subjetiva y satisfacción vital percibidas por los jóvenes con discapacidad intelectual.

Palabras clave: Discapacidad intelectual; Calidad de vida familiar; Bienestar subjetivo; Felicidad; Funcionamiento familiar.

\section{ABSTRACT}

The present study aimed to identify the relationship between the quality of Family Life, Family Functioning and Subjective Well-being, along with the factors of Quality of Family Life in relation to subjective well-being in young people with intellectual disabilities from a special education setting and work in the city of Talca (Chile). The sample consisted of 53 young people aged 16 to 24 years and 53 representatives, parents or guardians. All youth in the sample have been diagnosed with intellectual disabilities, of which $52.8 \%$ intellectual disability manifested sole predictor of their condition, while the remaining $47.2 \%$ corresponds to this co morbidity with other types of disability associated. The levels of this condition are ranging from mild reaching $37.7 \%$, followed by moderate $50.9 \%$, and finally severe and deep with $3.8 \%$. In order to measure the study variables they were administered four instruments: (1) Scale of Quality of Family Life (Verdugo, Sainz \& Rodriguez, 2009), (2) Family Functioning Questionnaire (Smilkstein, Ashworth, \& Montano, 1982), (3) The Satisfaction with Life Scale (Diener, Emmons, Larder \& Griffin, 1984), and (4) Subjective Happiness Scale (Lyubomirsky \& Lepper, 1999).

Considering previous studies, we formulated the following hypotheses: (1) At higher levels of life satisfaction, higher levels of subjective happiness in young people with intellectual disabilities, (2) there is a significant positive relationship between Family Functioning and Happiness Levels Subjective life satisfaction in young people with intellectual disabilities and (3) there are significant positive relationships between the factors of quality of family life and the components of subjective well-being in young people with intellectual disabilities.

For the study used a descriptive methodology and cross-sectional correlation seeking to establish concomitant relationships between variables. In relation to the variable quality of family life on two levels, importance and satisfaction, there is evidence that parents in the sample assigned more importance to the quality of family life to the satisfaction of it, because the parents at $49,1 \%$ assign more importance to the quality of family life in relation to $24.5 \%$ assigned to the satisfaction of the quality of family life. The women assigned greater importance to the quality of family life and satisfaction of this compared to men. With regard to family functioning, youths with intellectual disabilities normofunctionals consider the interactions of their respective families; those who have a moderate level of intellectual disability are those that show greater family functioning. Women perceive greater family functioning compared to men in the sample. With regard to subjective well-being into its two components, subjective happiness and life satisfaction, it appears that youths with intellectual disabilities remain a median subjective happiness and life satisfaction, feeling more satisfied than happy. It is observed that those with a moderate level of intellectual disability have a higher perception of subjective happiness and life sat- isfaction.

The main results show a significant relationship between the importance of the quality of family life satisfaction and the quality of family life $(r=.402 ; p<.01)$, between subjective happiness and life satisfaction $(r=.457: p<.01)$ and between subjective happiness and family functioning $(r=.388 ; p<.05)$. The factors of perceived quality of family life for parents: family interaction, parental role, physical and material, emotional and support for people with disabilities do not maintain a relationship with subjective happiness and life satisfaction perceived by young people with intellectual disabilities. 
Key words: Intellectual disability; Family life; Subjective well-being; Happiness; Family functioning.

Desde tiempos remotos, las personas con condiciones especiales han sufrido la discriminación y la exclusión social, sobre todo cuando se trata de discapacidad intelectual (Cordeu, 2008). Ahora bien, en Chile un $12.93 \%$ de la población presenta una discapacidad en cualquiera de sus grados, equivalente a 2.068.072 individuos, y en uno de cada tres hogares hay al menos un miembro con discapacidad. Dado este contexto, es relevante señalar que tanto los países como las instituciones involucradas deben planificar una inclusión social adecuada de estas personas para lograr un desarrollo eficiente y eficaz (Fondo Nacional de la Discapacidad, 2006). Este tipo de situaciones impide que los sujetos con discapacidad puedan desarrollar un papel relevante en el contexto social al cual pertenecen, generándose una brecha entre la limitación que presenta el individuo como tal y las capacidades que eventualmente podría adquirir y demostrar (Puga, 2005).

La discapacidad es una condición que se agrava aún más con las interacciones negativas que se dan entre la persona y su medio ambiente, incluyendo aspectos físicos, sociales y humanos (Zondek, Zepeda, González \& Recabarren, 2006a). El modelo clave en la discapacidad es la concepción socioecológica, que plantea que esta condición no depende única y exclusivamente de la persona que la padece, sino que es el resultado de la influencia de una serie de elementos de su entorno (Devlieger, Rusch \& Pfeiffer, 2003), apareciendo delimitado el rol de los apoyos que recibe desde los diferentes aspectos de su vida (Deaño, 2007; Powers, Dinerstein \& Holmes, 2005; Schalock, 2004; Vehmas, 2004).

La discapacidad se ha definido como una deficiencia o minusvalía, referida a una re- presentación social construida en base a imágenes mentales relacionadas con limitaciones y carencia de oportunidades de desarrollo humano y se ha concebido como un problema social. Su tratamiento se enfoca en dos sentidos: la persona con discapacidad y la comunidad en la que se inserta.

Para diagnosticar la discapacidad de una persona se deben investigar los siguientes puntos: si es deficiencia o daño, tipo de discapacidad, orígenes o causas, si es heredada o adquirida, capacidades residuales, posibilidades y tipos de rehabilitación (Zondek, Zepeda, González \& Recabarren, 2006). La Organización Mundial de la Salud (Zondek et al., 2006) define a la discapacidad como el resultado de la interacción entre la deficiencia de una persona y las variables ambientales que incluyen el ambiente físico, las situaciones sociales y los recursos humanos. Verdugo (1997a) menciona que la discapacidad es fluida, continua y cambiante, y que depende de las limitaciones funcionales que presenta la persona, ello también depende de los apoyos disponibles. Además, se puede proveer de servicios centrados en la conducta adaptativa y en su rol social, evaluando el nivel de reducción de dichas limitaciones (Schalock, 1998; Verdugo, 1997b).

Todas las personas a lo largo de su vida han tenido algún tipo de falta de capacidad al desarrollar funciones o actividades, ya sea de forma temporal, permanente, estable o creciente (Rodríguez-Porrero, 2000). García y Fernández (2005) mencionan que la discapacidad no se entiende como un proceso que tenga su origen en el individuo, sino más bien en procesos sociales, tales como: interacciones interpersonales e interinstitucionales y en las oportunidades que ofrece el medio. Por ello, diversos investigadores han tratado de aclarar cuáles son las dificultades más complejas al estudiar la calidad de vida de estos individuos que impiden su proceso de adaptación (Zondek et al., 2006).

La evaluación de la calidad de vida en familias con uno o más miembros con discapacidad es un tema central de los estudios interculturales (Verdugo, Córdoba \& Gó- 
mez, 2006), consensuando que su análisis no debería realizarse bajo una aproximación psicopatológica, sino más bien a través de una concepción en la que se prioricen las potencialidades y las capacidades de la familia (Turnbull, Blue-Banning, Turbiville \& Park, 1999; Turnbull, A.P. \& Turnbull, H.R., 2001).

Cabe destacar que las limitaciones de un individuo tienen directa relación con el impacto de la deficiencia que presenta junto con las condiciones del entorno construido y las del entorno social. Así, el nivel de funcionamiento que una persona presenta en su vida depende de aquellos elementos de compensación y ajuste que están a disposición a partir de dicho contexto (Deaño, 2007). Además, es relevante destacar que el constructo actual de discapacidad está centrado en la expresión de limitaciones con respecto al funcionamiento individual dentro de un contexto social, lo que repercute en el sujeto como una desventaja que se inicia como una condición de salud con resultados de déficit en el cuerpo y sus estructuras (Schalock et al., 2007).

Schalock y colaboradores (2005) demostraron que la calidad de vida familiar se puede analizar independientemente de aquella centrada en la persona. Por ello, la calidad de vida es considerada como un concepto multidimensional (Zondek et al., 2006)

Asimismo, contar con el apoyo familiar es fundamental en el desarrollo de la socialización de las personas con discapacidad, puesto que es la familia el sistema directo en el cual se adquieren las primeras interacciones humanas (Arés, 2009). En el caso de los jóvenes de la muestra, el apoyo de su núcleo familiar es fundamental para su desarrollo, dada la condición de discapacidad intelectual que comparten. Estas situaciones son las que llevan a que sea la propia persona quien elabore las respuestas, piense y sienta respecto a su vida, tanto a nivel cognitivo como a nivel afectivo (Cuadra \& Florenzano, 2003).

Establecer una estructura referente a los aspectos del ámbito de la educación especial es un tema complejo, ya que existe una serie de variables involucradas, tales como los profesionales, los padres y los individuos con discapacidad. De esta manera, el Centro de Investigación y Desarrollo de la Educación (CIDE, 1997, citado en Díaz, 2004) señala las siguientes dificultades en relación con la educación especial: (1) dificultad en la identificación del trastorno o discapacidad, (2) dificultad con los criterios e instrumentos de medida, son difícilmente transferibles de una situación a otra y (3) dificultad para obtener muestras representativas y generalizar los resultados.

De acuerdo al informe del CIDE (1998, citado en Díaz, 2004) en España se han presentado líneas de investigación en educación especial relacionadas con los siguientes temas: (1) sistemas especiales y situaciones de integración, (2) profesores y padres: comparación de modelos de trabajo; estudios de casos específicos de participación de padres de niños disminuidos y normales; estudios de casos específicos en el aula, (3) preocupación por el equipamiento en el ámbito escolar y (4) aspectos sociales de la experiencia en niños.

Las investigaciones que se han llevado a cabo en el área especializada tratan temáticas tales como: (1) las ventajas y desventajas de la integración, (2) la búsqueda de criterios relacionados con el diagnóstico, (3) la determinación de la validez de tratamientos y/o programas, (4) la descripción de las ventajas e inconvenientes de programas, (5) la evaluación de la eficacia de los programas y servicios sociales y (6) la medición de actitudes de la población general sobre la integración (Díaz, 2004).

Por último, es importante mencionar que en un estudio realizado en la Universidad de Las Palmas de Gran Canaria (Díaz, 2004) acerca de la percepción de los profesores hacia los alumnos con discapacidad, hay dos resultados relevantes: el primero indica que son las profesoras quienes se muestran más interesadas que los profesores en facilitar el acceso de los estudiantes con discapacidad de sus inmediaciones y el segundo muestra que los profesores, incluidos hombres y mujeres, 
que tienen o han tenido estudiantes con discapacidad en las aulas, dan a conocer que se les debe exigir a éstos los mismos contenidos que a los estudiantes capacitados.

El concepto de calidad de vida ha cobrado relevancia social como un indicador de éxito de los programas que generan prevención e intervención para personas con discapacidad (Córdoba-Andrade, GómezBenito \& Verdugo-Alonso, 2008).

Ahora bien, Giné (2004) plantea que ha surgido un gran interés por estudiar la calidad de vida, tanto desde un punto de vista personal, como también político y científico, ya que se asocia a situaciones de restricciones en la participación social.

Es así que Corraliza (1991) y posteriormente Ferreyra (2003) mencionan que la calidad de vida es un concepto que surge relacionado con el desarrollo de la sociedad de bienestar y que no sólo es visto como el grado de satisfacción subjetiva de un individuo, sino también como el nivel de recursos de los que dispone un individuo para poder dirigir su vida. Schalock (1999) menciona que el concepto de calidad de vida ha surgido bajo tres clasificaciones: (1) como un concepto de sensibilización, que aporta un punto de referencia y guía desde el sujeto, (2) como constructo social, usado como principio general para mejorar la calidad de vida de una persona y (3) como tema unificador, girando a su alrededor tanto el programa de rehabilitación como la evaluación del mismo.

En el ámbito de la salud mental, Verdugo y Martín (2002) mencionan que la calidad de vida como concepto se encuentra asociado a enfoques que tienen como objetivo mejorar las condiciones de vida de las personas con trastornos psicológicos, considerando de esta manera sus necesidades individuales. Es importante destacar que los elementos claves en la calidad de vida de las personas con discapacidad intelectual están asociados a la capacidad de toma de decisiones, autocontrol, independencia y autonomía (Arostegui, 1999).

Cabe destacar que hay dos estudios que dan cuenta de los efectos que tiene la disca- pacidad en los niveles de satisfacción de la persona, aunque con resultados contradictorios. Lucas (citado en Pagán-Rodríguez, 2010) muestra una inadaptación de la discapacidad en sujetos de Inglaterra y Alemania, mientras que Oswald y Powdthavee (2005 citados en Pagán-Rodríguez, 2010) obtuvieron una adaptación parcial, de un 30 a un $50 \%$, de acuerdo al grado de severidad de la discapacidad en países europeos. Smith y Alston (2009) dan a conocer que en algunos estudios se muestra que la relación entre discapacidad física y satisfacción vital ha llegado a ser inconsistente, producto del alto nivel de los juicios negativos que afectan a la satisfacción. Además, una alta correlación de la tasa de bienestar subjetivo personal con una variedad de mediciones físicas, es capaz de aumentar la vida de la persona, la calidad del sueño, felicidad, sociabilidad y extraversión (Pagán-Rodríguez, 2010).

De esta manera, surge la necesidad de profundizar acerca de qué tan importante y satisfactoria es la calidad de vida que reciben en sus hogares los jóvenes con discapacidad intelectual de la muestra, considerando que hay estudios que indican que un número considerable de las familias que tienen uno o más miembros con discapacidad, actúa de forma silenciosa, sin solicitar la ayuda necesaria (Puga, 2005).

Las familias de las personas con discapacidad se diferencian en sus características de aquellas que no tienen esta condición dentro de su núcleo, requiriendo atención psicosocial y orientación profesional para lograr una dinámica más armónica y positiva que favorezca el desarrollo de la persona con discapacidad (Valls, Vilá \& Pallisera, 2004). Así, la calidad de vida de la familia se asocia al bienestar personal que va más allá de lo individual, alcanzando así a la comunidad (Mora, Córdoba \& Bedoya, 2007).

Además, dado que las personas con discapacidad tienen una participación social limitada, hay más elementos riesgosos que resultan como amenazas para su calidad de vida (Giné, 2004).

En el estudio que se informa se analizó la relación entre calidad de vida familiar, fun- 
cionamiento familiar y bienestar subjetivo en jóvenes con discapacidad intelectual de un establecimiento de educación especial y laboral.

\section{MÉTOdO}

\section{MUESTRA}

La muestra seleccionada fue probabilística por conveniencia, y sus participantes fueron elegidos porque cumplían con los requisitos del estudio, ya que eran jóvenes diagnosticados con discapacidad intelectual y sus tutores, quienes representaban el núcleo de su familia. Fueron 53 alumnos (de 16 a 24 años de edad) y 53 apoderados (padres o tutores) de un establecimiento de educación especial y laboral de la ciudad de Talca. Un $52.8 \%$ de los jóvenes manifestó la discapacidad intelectual como único predictor de su condición.

\section{INSTRUMENTOS}

Se aplicaron cuatro instrumentos:

1.- La Escala de Calidad de Vida Familiar (Verdugo, Sainz \& Rodríguez, 2009) que contiene 41 ítemes agrupados en cinco factores y son los siguientes: interacción familiar, rol parental, bienestar físico y material, bienestar emocional y apoyo para personas con discapacidad. La escala tiene estabilidad temporal adecuada, con coeficiente de la escala global, tanto para la importancia (.68) como para el grado de satisfacción (.78).

2.- La versión traducida al español (Be1lon, Delgado, Luna del Castillo \& Lardelli, 1996) del Family Functioning Questionnaire (Smilkstein, Ashworth \& Montano, 1982), compuesto por cinco ítemes con tres opciones de respuesta, cuya interpretación corresponde a tres clasificaciones: normo-funcional, disfunción leve y disfunción grave.

3.- La adaptación y validación de Moyano y Ramos Alvarado (2007) de la Satisfaction with the Life Scale (Diener, Emmons, Larden \& Griffin), cuyo objetivo es la me- dición de la satisfacción con la vida mediante un juicio cognitivo global que hace sobre su vida quien responde. Está compuesta por cinco ítemes, que se responden a través de una escala tipo Likert, donde 1 es muy en desacuerdo y 7, muy de acuerdo.

4.- La versión chilena (Moyano-Díaz \& Ramos, 2007) de la Subjective Happiness Scale (Lyubomirsky \& Lepper, 1999), compuesta por cuatro ítemes, que se responden de acuerdo a una escala de diferencial semántico de 1 a 7 , donde 7 representa la mayor felicidad.

\section{PROCEDIMIENTO Y TRATAMIENTO DE LOS DATOS}

Luego de contar con la autorización de las autoridades del establecimiento de educación se realizó un consentimiento informado, asegurando el anonimato de quienes responderían.

Los cuatro instrumentos fueron entregados y retirados durante las 2 semanas de aplicación. La Escala de Calidad de Vida Familiar fue respondida por los tutores y los estudiantes respondieron las otras puebas.

Los datos fueron procesados y analizados utilizando el programa SPSS 15.0, mediante el cual se realizaron los análisis descriptivos y correlacionales (correlación de Pearson o Rho de Spearman según la prueba de normalidad K-S realizada con cada variable).

\section{Resultados}

Se presentan los análisis derivados de la investigación. En primer lugar, los análisis descriptivos de la Calidad de Vida Familiar, Bienestar Subjetivo yFuncionamiento $\mathrm{Fa}$ miliar y luego los análisis correlacionales entre Calidad de Vida Familiar, Bienestar Subjetivo y Funcionamiento Familiar.

Cabe destacar que en las tablas se informan valores perdidos iguales a 13 y que dan como muestra un $n=40$, los mismos corresponden a estudiantes cuya discapacidad les impidió contestar los cuestionarios, pero 
sus tutores sí respondieron, por ende, se incluyeron las respuestas de los padres, pero no así la de los hijos dada su condición.

En la Tabla 1 se observan las medias y los desvíos típicos de Importancia de la Calidad de Vida Familiar (ICVF) y de Satisfacción de la Calidad de Vida Familiar (SCVF). Para la primera, existen 20 casos de discapacidad leve $(M=110.9 ; D E=16.3), 2$ en el nivel profundo $(M=100.5 ; D E=34.7), 27$ en el nivel moderado $(\mathrm{M}=116.4 ; \mathrm{DE}=11.9), 2$ en el nivel desconocido $(M=116 ; D E=12.7)$ y 2 en el nivel severo $(M=123.5 ; D E=2,1)$. Se muestran a su vez, los resultados de ICVF $(M=114.0 ; D E=14.5)$. En cuanto a la SCVF, existen 20 casos en discapacidad leve $(M=95.5 ; D E=18.3), 2$ en el nivel profundo $(\mathrm{M}=74.0 ; D E=5.7), 27$ en el nivel moderado $(M=97.2 ; D E=19.5), 2$ en el nivel desconocido $(M=106 ; D E=4.2)$ y 2 en el nivel severo $(M=102.5 ; D E=19.1)$. Se muestran también los resultados de SCVF $(M=96.2 ; D E=18.6)$.

En la Tabla 2 se informa el Funcionamiento Familiar $(M=6.2 ; D E=2.6)$ en 16 casos con discapacidad leve $(M=5.9$; $D E=2.9), 1$ en el nivel profundo $(M=6.0$; sin desviación), 20 en el nivel moderado $(M=6.4 ; \mathrm{DE}=2,5), 2$ en el nivel desconocido $(M=7.5 ; D E=.7)$ y 1 en el nivel severo $(M=4.0 ;$ sin desviación). Se observaron 28 casos de Funcionamiento Familiar en hombres $(M=6.2 ; D E=2.6)$ y 12 en mujeres $(M=6.4 ; D E=2.7)$.

En la Tabla 3 se observan las dos dimensiones del Bienestar Subjetivo: Felicidad Subjetiva (FS) $(M=16.8 ; D E=6.4)$ y Satisfacción Vital (SV) $(M=18.8 ; D E=8.9)$. La primera aparece en 16 casos en el nivel de discapacidad leve $(M=16.7 ; D E=5.8)$, 1 en el nivel profundo $(M=12.0$; sin desviación), 20 en el nivel moderado $(M=17.3$; $D E=7.3), 2$ en el nivel desconocido $(M=19.0 ; D E=1.4)$ y 1 en el nivel severo $(M=11.0 ;$ sin desviación). La segunda se observa en 16 casos en el nivel leve de discapacidad $(M=17.8 ; D E=7.9) ; 1$ en el nivel profundo $(M=12.0 ;$ sin desviación $), 20$ en el nivel moderado $(M=19.1 ; D E=9.7)$, 2 en el nivel desconocido $(M=19.0$;
$D E=7.1)$ y 1 en el nivel severo $(M=35.0$; sin desviación).

Con respecto a los niveles de Felicidad Subjetiva y Satisfacción Vital, se observan 28 casos de FS $(M=17.8 ; D E=6.4)$ y de SV $(M=19.3 ; D E=9.3)$ en hombres y 12 casos de FS $(M=14.7 ; D E=6.1)$ y de SV $(M=$ $17.7 ; D E=8.4)$ en mujeres. Asimismo, existen 7 casos de una baja FS (13.2\%), 27 de mediana FS $(50.9 \%)$ y 6 de alta FS $(11.3 \%)$ de un total de 40 equivalentes al $75.5 \%$ de la muestra total. Se reportan 13 casos perdidos en total $(24.5 \%)$. Con respecto a SV, 15 casos corresponden a una baja SV (28.3\%), 16 a una mediana SV $(30.2 \%)$ y 9 a una alta SV $(17.0 \%)$, siendo la muestra total de 40 jóvenes. Se reportan 13 casos perdidos en total $(24.5 \%)$.

En la Tabla 4 se observa que existe correlación significativa entre Importancia de la Calidad de Vida Familiar y Satisfacción de la Calidad de Vida Familiar $(r=.402$; $p<.01$ ); entre Felicidad Subjetiva y Satisfacción Vital $(r=.457 ; p<.01)$ y entre Felicidad Subjetiva y Funcionamiento Familiar $(r=.388 ; p<.05)$. Con respecto a la relación entre los factores de Calidad de Vida Familiar y Bienestar Subjetivo no se observan relaciones significativas, por lo cual no aparecen en la Tabla $4(r=.14 ; r=.114$; $r=-.06 ; r=.08 ; r=.18 ; r=.17 ; r=-.02$; $r=.02 ; r=-.7 ; r=-.99 ; p<.01)$.

\section{DISCUSIÓN Y CONCLUSIONES}

Cabe destacar considerando los resultados obtenidos en el estudio, que la tradición del bienestar subjetivo presente en los jóvenes de la muestra es heudaimónica (Ryan \& Deci, 2001), puesto que dichos individuos no consideran la felicidad como el principal criterio para elaborar sus pensamientos con respecto a la situación de su propia vida a partir de su condición de discapacidad intelectual, sino más bien manifiestan que les genera mayor recompensa personal en dicha elaboración sentirse satisfecho tal como son, a pesar de las dificultades asociadas a los diferentes niveles de discapacidades que 
presentan (Barrientos, 2005; Carruthers \& Deyell Hood, 2004; Diener, 2000; Ryan \& Deci, 2001; Ryff, Singer \& Dienberg, 2004; Villar, Resano, Osuna \& Triadó, 2003).

En concordancia con la idea anterior, los jóvenes del estudio manifiestan niveles de satisfacción vital que son directamente proporcionales con los de felicidad subjetiva, puesto que si bien es mayor el primer componente del bienestar subjetivo por sobre el segundo, la distribución de los datos es similar en ambas variables. Este hecho se contradice con lo planteado por Veenhoven (1994), quien menciona que el bienestar subjetivo de las personas con discapacidad se ve disminuido por su condición.

De esta manera, un elemento relevante es que de los jóvenes que conforman la muestra, los hombres presentan mayores niveles de SV y de FS en comparación con las mujeres. Esto se contradice con lo propuesto por Inglehart (2002) acerca de que las mujeres durante la adolescencia presentan niveles mayores de bienestar subjetivo.

Ahora bien, la familia es el núcleo responsable para generar niveles de funcionamiento y calidad de vida familiar estables y funcionales en las personas, sobre todo en los jóvenes de la muestra, puesto que cuanto mejores sean sus interacciones familiares, mayor será su nivel de calidad de vida y felicidad; mientras que ocurrirá lo contrario si las relaciones en sus hogares presentaran problemas y elementos más bien negativos, como un bajo apoyo y consenso familiar ante las exigencias que representa tener un integrante con discapacidad (Mora et al., 2007).

Con respecto a la idea anterior, es relevante dejar en claro que las familias de los jóvenes de la muestra comparten la característica de ser un pilar fundamental en el desarrollo y crecimiento personal de ellos, esto puede deberse al amor incondicional que tienen hacia sus miembros con capacidades especiales.

Asimismo, existen algunos elementos que son importantes a considerar en futuras investigaciones que relacionen la discapacidad con la calidad de vida familiar y el bienestar subjetivo, entre ellos la necesidad de aumentar el número de la muestra, con el fin de fortalecer en mayor medida los resultados. En relación a los instrumentos utilizados, éstos fueron adecuados. Otro punto a considerar son otras variables que podrían ser consideradas en próximos estudios, tales como la inserción laboral y el apoyo social. Además, es importante considerar en futuras investigaciones el grado certero de discapacidad, los estándares de los niveles y las políticas públicas asociadas.

Así, para reconocer a una persona como discapacitada se debe investigar si es una deficiencia o un daño, el tipo de discapacidad, cuáles son sus orígenes o causas, si es heredada o adquirida, las capacidades residuales, las posibilidades y los tipos de rehabilitación, entre otras (Zondek et al., 2006).

En cuanto a la educación especial de las personas con condición de discapacidad, existen dificultades en su clasificación debido a que los criterios e instrumentos de medición son difícilmente transferibles de una situación a otra, lo cual limita las posibilidades de comparar resultados y la dificultad para obtener muestras representativas en cuanto al número como al tipo de discapacidad (Díaz, 2004). 
TABLA 1

MEDIAS Y DESVIOOS TíPICOS DE LA CALIDAD DE VIDA FAMILIAR EN FUNCIÓN DEL NIVEL DE DISCAPACIDAD EN JÓVENES

\begin{tabular}{|c|c|c|c|c|c|c|}
\hline \multirow{2}{*}{$\begin{array}{c}\text { Nivel de } \\
\text { discapacidad }\end{array}$} & \multicolumn{3}{|c|}{ Importancia } & \multicolumn{3}{|c|}{ Satisfacción } \\
\hline & $M$ & $D T$ & $n$ & $M$ & $D T$ & $n$ \\
\hline Leve & 110.9 & 16.3 & 20 & 95.5 & 18.3 & 20 \\
\hline Profundo & 100.5 & 34.7 & 2 & 74.0 & 5.7 & 2 \\
\hline Moderado & 116.4 & 11.9 & 27 & 97.2 & 19.5 & 27 \\
\hline Desconocida & 116.0 & 12.7 & 2 & 106.0 & 4.2 & 2 \\
\hline Severa & 123.5 & 2.1 & 2 & 102.5 & 19.1 & 2 \\
\hline Total & 114.0 & 14.5 & 53 & 96.2 & 18.6 & 53 \\
\hline
\end{tabular}

TABLA 2

MEDIAS Y DESVIOOS TíPICOS DEL FUNCIONAMIENTO FAMILIAR RELACIONADO CON EL NIVEL DE DISCAPACIDAD

\begin{tabular}{l|cc|c}
\hline \multirow{2}{*}{$\begin{array}{c}\text { Nivel de } \\
\text { discapacidad }\end{array}$} & $M$ & $D T$ & $n$ \\
\hline \multirow{2}{*}{ Leve } & 5.9 & 2.9 & 16 \\
Profundo & 6.0 & - & 1 \\
Moderado & 6.4 & 2.5 & 20 \\
Desconocida & 7.5 & 0.7 & 2 \\
Severa & 4.0 & - & 1 \\
& & & 40 \\
\hline \multirow{2}{*}{ Total } & 6.2 & 2.6 & 4 \\
\end{tabular}




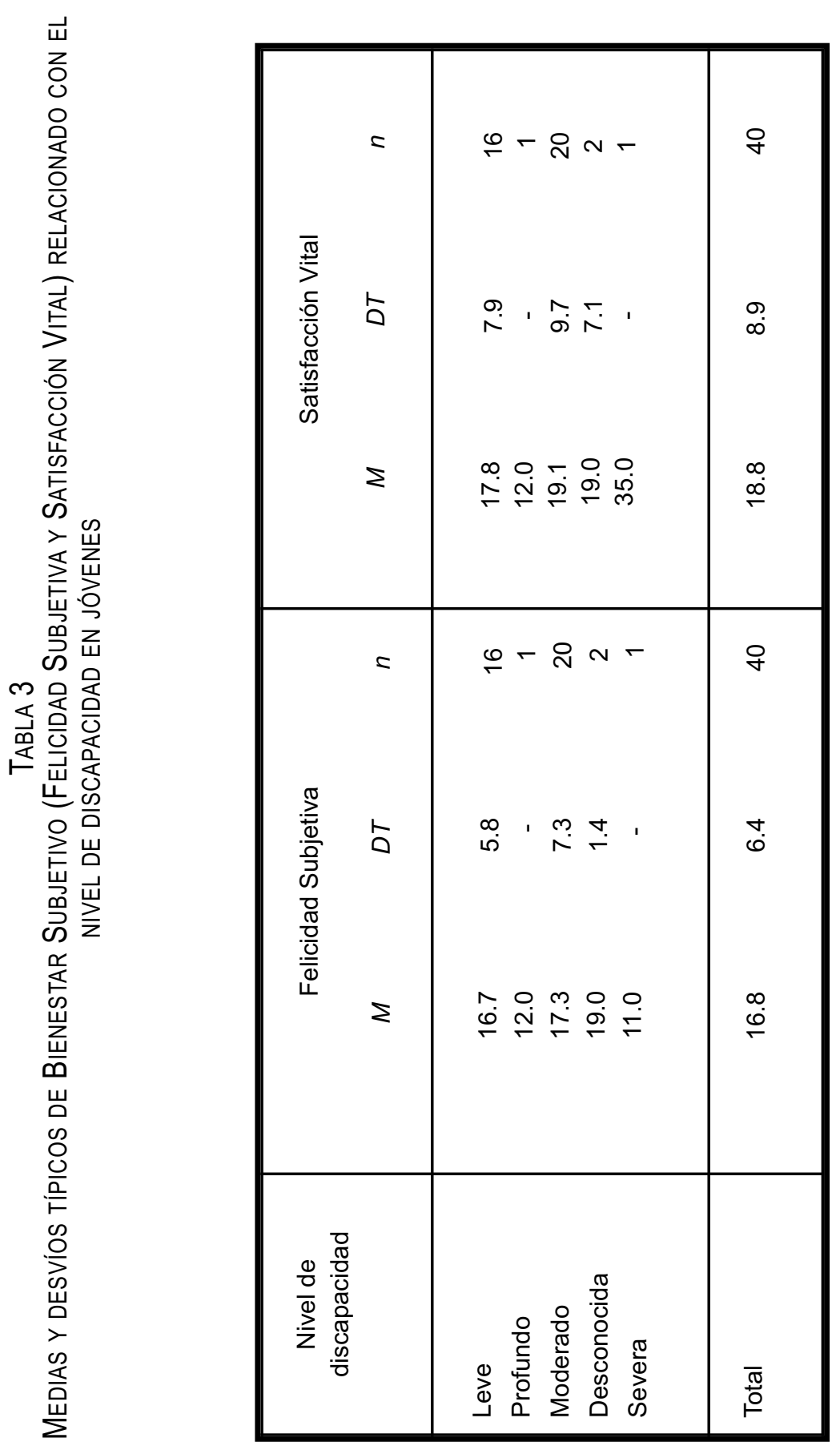




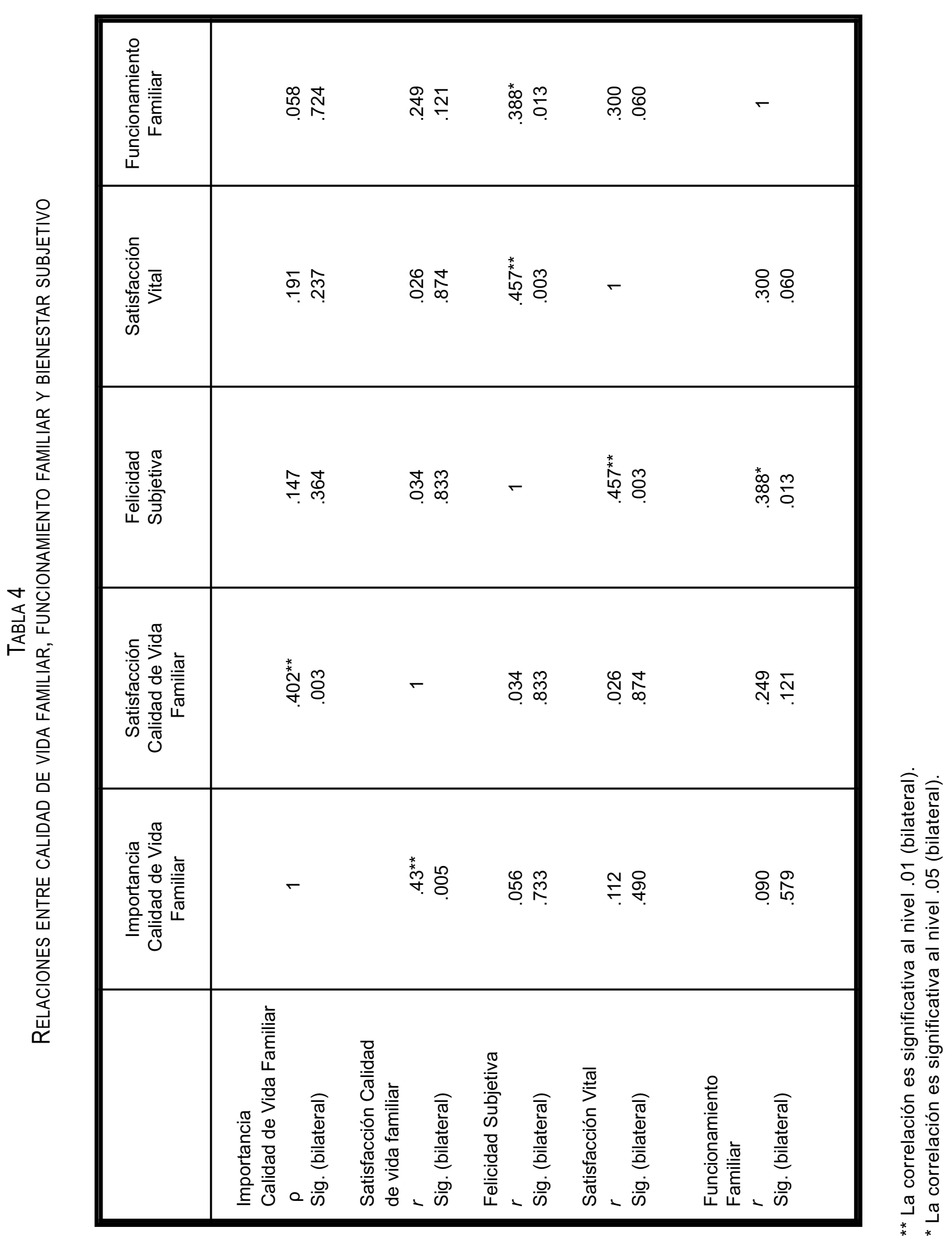




\section{REFERENCIAS BIBLIOGRÁFICAS}

Arés, P. (2009). Psicología de la familia en países latinos del siglo XXI [Family Psychology in XXI century Latin countries]. México: Asociación Mexicana de Alternativas en Psicología.

Arostegui, I. (1999). Evaluación de la calidad de vida en personas adultas con retraso mental [Assessing of the quality of life in adults with mental retardation]. Actas de las III Jornadas Cientificas de Investigación sobre Personas con Discapacidad, 405-414.

Barrientos, J. (2005). Calidad de vida, bienestar subjetivo: Una mirada psicosocial [Quality of life, subjetive well being: A psicosocial review]. Chile: Editorial Imprenta Salesianos.

Bellon, J.A, Delgado, A., Luna del Castillo \& J.D., Lardelli (1996). Validez y fiabilidad del Cuestionario de Función Familiar Apgar-familiar [Validity and fiability of the Familiar Function Apgar-familiar Questionnaire]. Atención Primaria, 18(6), 289-296.

Carruthers, C. \& Deyell Hood, C. (2004). The power of the positive: Leisure and well-being. Journal of Leisure Research, 38, 225-246.

Córdoba-Andrade, L., Gómez-Benito, J. \& Verdugo-Alonso, M. (2008). Calidad de vida familiar en personas con discapacidad: Un análisis comparativo [Family quality of life for people with disabilities: A comparative analysis]. Universitas Psychologica, 7(2), 369-383.

Cordeu, C. (2008). Reflexiones en torno a la inclusión socio-comunitaria de personas con discapacidad intelectual: La voz de sus protagonistas [Reflections on social and community inclusion of people with intellectual disabilities: the voices of their protagonists]. Tesis de maestría en Psicología. Universidad de Chile.
Corraliza, J.A. (1991). Ciudad, arquitectura y calidad de vida. Notas para una discusión [City, architecture and quality of life. Notes for a discussion]. Sevilla, España: Editorial Arquetipo.

Cuadra, H. \& Florenzano, R. (2003). El bienestar subjetivo: Hacia una psicología positiva [Subjective well-being: Toward a positive Psychology]. Revista de Psicología de la Universidad de Chile, 12(1), 83-96.

Deaño, M. (2007). Formación y discapacidad: La necesaria colaboración de la universidad [Education and disability: The need for collaboration of the University]. Departamento de Psicología Evolutiva y Comunicación. Universidad de Vigo.

Devlieger, J.P., Rusch, F. \& Pfeiffer, D. (2003). Rethinking disability: The emergence of new definition, concepts and communities. Antwerp, Belgium: Garant.

Díaz, G. (2004). Los estudiantes con discapacidad en la Universidad de Las Palmas de Gran Canaria [Students with disabilities at the University of Las Palmas de Gran Canaria]. España: Editorial Cometa.

Diener, E. (2000). Subjective well-being. The science of happiness and proposal for a National Index. American Psychologist, 55, 34-43. doi:10.1037//0003-066X.55.1.34.

Diener, E., Emmons, R., Larden, R. \& Griffin, S. (1984). The Satisfaction with the Life Scale. Journal of Personality Assessment, 49, 71-75. doi:10.1037//00223514.47.3.580.

Ferreyra, S. (2003). La calidad de vida como concepto. Su utilización en el accionar de los trabajadores sociales en el ámbito de la salud [The quality of life as a concept. Its use in the actions of social workers in the field of health]. Revista Servicio Social y Sociedade, 74. Brasil.

FONADIS - Fondo Nacional de la Discapacidad (2006). Encuesta Casen 2006 [National 
Socioeconomic Characterization]. Santiago: Gobierno de Chile, Ministerio de Planificación.

García, A. \& Fernández, A. (2005). La inclusión para las personas con discapacidad: Entre la igualdad y la diferencia [The inclusion for people with disabilities: Between equality and difference]. Revista Ciencias de la Salud / Bogotá, 3(2), 235-246.

Giné, C. (2004). Servicios y calidad de vida para las personas con discapacidad intelectual [Services and quality of life for people with intellectual disabilities]. Revista Española sobre Discapacidad Intelectual, 35(2), 1-14.

Inglehart, R. (2002). Gender, aging and subjective well-being. International Journal of Comparative Sociology, 43, 391-408. doi:10. 1177/002071520204300309.

Lyubomirsky, S. \& Lepper, H. (1999). A measure of subjective happiness: Preliminary reliability and construct validation. Social Indicators Research, 46(2), 137-156. doi:10.1023/A:100 6824100041.

Mora, A., Córdoba, L. \& Bedoya, A. (2007). Familias de adultos con discapacidad intelectual en Cali, Colombia, desde el modelo de calidad de vida [Families of adults with intellectual disability in Cali, Colombia, from the quality of life model]. Revista Psykhe, 16(2), 29-42.

Moyano-Díaz, E. \& Ramos Alvarado, N. (2007). Bienestar subjetivo: Midiendo satisfacción vital, felicidad y salud en población chilena de la Región Maule [Subjective well-being: Measuring life satisfaction, happiness and health in Chilean population of Maule Region]. Revista Universum, 22(2), 177-193. doi: 10.40 67/S0718-23762007000200012.

Pagán-Rodríguez, R. (2010). Onset of disability and life satisfaction: Evidence from the German socio-economic panel. The European
Journal Health Economics, 11, 471-485. doi: 10.1007/s10198-009-0184-z.

Powers, L., Dinerstein, R. \& Holmes, S. (2005). Self-advocacy, self-determination, social freedom and opportunity. En K.C. Lakin \& A. Turnbull (Eds.), National goals and research for people with intellectual and developmental disabilities (pp. 257-287). Washington, DC: American Association on Mental Retardation.

Puga, D. (2005). La dependencia de las personas con discapacidad: Entre lo sanitario y lo social, entre lo privado y lo público [The dependence of people with disabilities: health and between the social, between the private and the public]. Revista Española de Salud Pública, 79, 327-330.

Rodríguez-Porrero, C. (2000). Discapacidad y calidad de vida [Disability and quality of life]. Revista Multidisciplinar de Gerontología, 10(2), 66-91.

Ryan, R. \& Deci, E. (2001). On happiness and human potentials: A review of research on hedonic and eudemonic well-being. Annual Review of Psychology, 52, 141-166. doi: 10. 1146/annurev.psych.52.1.141

Ryff, C., Singer, B. \& Dienberg, G. (2004). Positive health: Connecting well-being with biology. The Royal Society, 359, 1383-1394. doi:10.1098/rstb.2004.1521.

Schalock, R.L. (1998). The assessment and rehabilitation of persons with traumatic brain injury. Applied and Preventive Psychology, 7(4), 247-253. doi:10.1016/S09621849(98) 80028-0.

Schalock, R. (1999). Hacia una nueva concepción de la discapacidad [Towards a new conception of disability]. III Jornadas Científicas de Investigación sobre Personas con Discapacidad. Universidad de Salamanca. España. Schalock, R.L. (2004). The emerging disability paradigm and its implications for policy and 
practice. Journal of Disability Policy Studies, 14, 204-215. doi:10.1177/10442073040 140040201.

Schalock, R.L., Luckasson, R., Shogren, K., Borthwick-Duffy, S., Bradley, V., Buntinx, W. et al. (2007). El nuevo nombre del retraso mental: Comprendiendo el cambio al término discapacidad intelectual [The new name of mental retardation: understanding the change to the term intellectual disability]. Siglo Cero, 38(4), 1-19.

Schalock, R.L., Verdugo, M.A., Jenaro, C., Wang, M., Wehmeyer, M., Xu, J. \& Lachapelle, Y. (2005). Cross-cultural study of quality of life indicators. American Journal on Mental Retardation, 110(4), 298-311. doi:10.1111/j.13 6527 88.2005.00742.x.

Smilkstein, G., Ashworth, C. \& Montano, D. (1982). Validity and reliability of the Family APGAR as a test of family function. The Journal of Family Practice, 15, 303-311.

Smith, D. \& Alston, R. (2009). The relationship of race and disability to life satisfaction in the United States. Journal of Rehabilitation, 75(1), 3-9.

Turnbull, A.P., Blue-Banning, M., Turbiville, V. \& Park, J. (1999). From parent education to partnership education: A call for a transformed focus. Topics in Early Childhood Special Education, 19(3), 164-171. doi:10.1 77/027112149901900308

Turnbull, A.P. \& Turnbull, H.R. (2001). Families, professionals, and exceptionality: Collaborating for empowerment (4ta. ed.). Upper Saddle River, NJ: Merrill Prentice Hall.

Valls, M., Vilá, M. \& Pallisera, M. (2004). La inserción de las personas con discapacidad en el trabajo ordinario: El papel de la familia [The inclusion of people with disabilities in ordinary work: The role of the family]. Revista de Educación, 334, 99-117.
Veenhoven, R. (1994). El estudio de la satisfacción con la vida [The study of life satisfaction]. Intervención Psicosocial, 3, 87-116.

Vehmas, S. (2004). Dimensions of disability. Cambridge Quarterly of Healthcare Ethics, 13, 34-40. doi:10.1017/S096318010 41310 71.

Verdugo, M.A. (1997a). III Jornadas Científicas de Investigación sobre Personas con Discapacidad. [III Scientific Meeting about Persons with Discapacity]. Universidad de Salamanca, España.

Verdugo, M.A. (mayo 1997b). A review of research published from 1991 to 1995 in 12 journals on research on mental retardation. Keynote address. Trabajo presentado en el 121st AAMB Annual Meeting, New York City.

Verdugo, M.A. (2002). Análisis de la definición de discapacidad intelectual de la Asociación Americana sobre Retraso Mental de 2002 [Analysis of the definition of intellectual disability of the American Association on Mental Retardation]. Revista Española sobre Discapacidad Intelectual, 34(1), 1-17.

Verdugo, M.A., Córdoba, L. \& Gómez, J. (2006). Adaptación y validación al español de la Escala de Calidad de Vida Familiar [Spanish adaptation and validation of the Family Quality of Life Survey]. Revista Española sobre Discapacidad Intelectual, 37 (218), 41-46.

Verdugo, M. \& Martín, M. (2002). Autodeterminación y calidad de vida en salud mental: Dos conceptos emergentes [Self-determination and quality of life in mental health: Two emerging concepts]. Revista de Salud Mental, 25(4), 68-77.

Verdugo, M.A., Sainz, F. \& Rodríguez, A. (2009). Escala de Calidad de Vida Familiar [Family Quality of Life Survey]. Salamanca: INICO. 
Villar, F., Resano, C., Osuna, J. \& Triadó, C. (2003). La medida del bienestar en personas mayores: Adaptación de la Escala RYFF [The welfare measure in older people: adaptation of the Ryff Scale]. Revista de Psicologia General y Aplicada, 58, 347-364.
Zondek, A., Zepeda, M., González, F. \& Recabarren, E. (2006). Discapacidad en Chile: Pasos hacia un modelo integral de funcionamiento humano [Disabilities in Chile: Steps towards a comprehensive model of human functioning]. Santiago: Maval.

$$
\begin{array}{r}
\text { Facultad de Psicología } \\
\text { Universidad de Talca } \\
\text { Avda. Lircay s/n } \\
\text { Talca - Chile }
\end{array}
$$

Fecha de recepción: 6 de junio de 2011 Fecha de aceptación: 23 de julio de 2012 
